\title{
Specific staphylococcal cassette chromosome mec (SCCmec) types and clonal complexes are associated with low-level amoxicillin/clavulanic acid and cefalotin resistance in methicillin-resistant Staphylococcus pseudintermedius
}

\begin{abstract}
Alice Wegener ${ }^{1}$, Peter Damborg ${ }^{2}$, Luca Guardabassi ${ }^{2,3}$, Arshnee Moodley $^{2}$, Lapo Mughini-Gras ${ }^{4,5}$, Birgitta Duim ${ }^{1}$, Jaap A. Wagenaar ${ }^{1,6}$ and Els M. Broens $\mathbb{D}^{1 *}$

${ }^{1}$ Department of Infectious Diseases and Immunology, Faculty of Veterinary Medicine, Utrecht University, Utrecht, The Netherlands;

${ }^{2}$ Department of Veterinary and Animal Sciences, Faculty of Health and Medical Sciences, University of Copenhagen, Frederiksberg, Denmark; ${ }^{3}$ Department of Pathobiology and Population Sciences, The Royal Veterinary College, North Mymms, UK; ${ }^{4}$ Center for Infectious Disease Control, National Institute for Public Health and the Environment (RIVM), Bilthoven, The Netherlands; ${ }^{5}$ Institute for Risk Assessment Sciences, Utrecht University, Utrecht, The Netherlands; ${ }^{6}$ Wageningen Bioveterinary Research, Lelystad,
\end{abstract} The Netherlands

*Corresponding author. E-mail: E.M.Broens@uu.nl

Received 24 July 2019; returned 19 September 2019; revised 1 November 2019; accepted 8 November 2019

Background: Staphylococcus pseudintermedius is a common pathogen in dogs and methicillin resistance has emerged over recent decades. According to the current guidelines, S. pseudintermedius displaying oxacillin resistance should be reported as resistant to all $\beta$-lactams.

Objectives: To identify possible associations between $\beta$-lactam resistance levels and clonal complexes (CCs) and/or staphylococcal cassette chromosome mec (SCCmec) types in methicillin-resistant S. pseudintermedius (MRSP).

\begin{abstract}
Methods: MICs of oxacillin, penicillin, ampicillin, amoxicillin/clavulanic acid and cefalotin were determined by broth microdilution for 86 clinical canine MRSP isolates from Denmark and the Netherlands. PCR and sequencing were used for SCCmec typing and MLST.
\end{abstract}

Results: Isolates belonged to CC71 $(n=36)$, CC258 $(n=33)$, CC45 $(n=11)$, CC68 $(n=1)$ and five singleton STs. SCCmecII-III was exclusively found in CC71 and SCCmecIV was significantly associated with CC258. SCCmecV and non-typeable SCCmec types occurred in 4 and 14 isolates, respectively. SCCmecIV was associated with lower MICs of oxacillin ( $<2 \mathrm{mg} / \mathrm{L})$, ampicillin $(<8 \mathrm{mg} / \mathrm{L})$ and amoxicillin/clavulanic acid $(<4 \mathrm{mg} / \mathrm{L})$ and with susceptibility to cefalotin $(<4 \mathrm{mg} / \mathrm{L})$. All isolates harbouring SCCmecV were susceptible to cefalotin as well.

Conclusions: $\mathrm{SCCmec}$ types were associated with different $\mathrm{CCs}$ and with either high- or low-level resistance to different $\beta$-lactams. The finding of amoxicillin/clavulanic acid (20\%) and cefalotin (70\%) in vitro susceptibility across all CCs might have clinical implications, since amoxicillin/clavulanic acid and first-generation cephalosporins are first-choice antibiotics for treatment of S. pseudintermedius infections. Pharmacokinetic/pharmacodynamic and clinical outcome studies are warranted to evaluate the in vivo efficacy of these $\beta$-lactams for treatment of MRSP infections.

\section{Introduction}

Staphylococcus pseudintermedius is an opportunistic pathogen in dogs. Methicillin-resistant S. pseudintermedius (MRSP) has emerged worldwide over the past decade. ${ }^{1,2}$ Methicillin resistance is associated with mecA located on staphylococcal cassette chromosome mec (SCCmec). ${ }^{3}$ Various $\mathrm{SCCmec}$ types in
Staphylococcus aureus are linked to different lineages, exhibiting different levels of oxacillin resistance. ${ }^{4-6}$ In S. pseudintermedius, similar associations have been suggested. ${ }^{7,8}$

Screening for methicillin resistance in $S$. pseudintermedius is performed by oxacillin susceptibility testing using a considerably lower resistance breakpoint $(R \geq 0.5 \mathrm{mg} / \mathrm{L}$ ) than for $S$. aureus 
( $\mathrm{R} \geq 4 \mathrm{mg} / \mathrm{L}$ ). ${ }^{9}$ Oxacillin-resistant isolates should be reported as resistant to all $\beta$-lactams according to an expert rule established for S. aureus to minimize major errors of antimicrobial susceptibility testing (i.e. resistant strains reported as susceptible). This rule was adapted to S. pseudintermedius on the basis of the oxacillin MIC distribution for mecA-positive isolates without being corroborated by any clinical outcome data. ${ }^{10}$

This study aimed to detect possible associations between $\beta$-lactam resistance levels and clonal complexes (CCS) and/or SCCmec types in MRSP. In vitro susceptibility to $\beta$-lactams commonly used in veterinary medicine was evaluated in a collection of clinical MRSP isolates that were fully characterized by SCCmec typing and MLST.

\section{Materials and methods}

This study included 86 canine clinical MRSP (2008-14) isolates previously described in the Netherlands $(n=50)$ and Denmark $(n=36)^{2,11,12}$ For Danish isolates, MLST was performed by PCR and Sanger sequencing and SCCmec types were determined by PCR. Dutch isolates were characterized by WGS using the batchupload pipeline from the Center for Genomic Epidemiology (Lyngby, Denmark) to assign multilocus STs and SCCmecFinder for SCCmec typing. ${ }^{13}$ When no SCCmec type was detected, a local BLASTn search was performed against SCCmec elements previously identified in S. pseudintermedius using Geneious version 2019.0.3 (Biomatters, Auckland, New Zealand).

MICs of five $\beta$-lactams (oxacillin range $0.5-2 \mathrm{mg} / \mathrm{L}$, penicillin range $0.5-8 \mathrm{mg} / \mathrm{L}$, ampicillin range $0.25-8 \mathrm{mg} / \mathrm{L}$, amoxicillin/clavulanic acid range $0.25-8 \mathrm{mg} / \mathrm{L}$ and cefalotin range $2-4 \mathrm{mg} / \mathrm{L}$ ) were determined by broth microdilution using commercially available systems: Sensititre (Thermo Fisher Scientific, Hvidovre, Denmark) in Denmark; and MICRONAUT (MERLIN Diagnostika GmbH, Germany) in the Netherlands. Quality control (S. aureus ATCC 29213) was used in both laboratories and resistance was based on veterinary clinical breakpoints according to CLSI. ${ }^{9}$

Resistance was classified as high- or low-level to facilitate statistical analysis. Isolates displaying MICs of oxacillin $\geq 2 \mathrm{mg} / \mathrm{L}$, of penicillin or ampicillin $\geq 8 \mathrm{mg} / \mathrm{L}$ and of amoxicillin/clavulanic acid $\geq 4 \mathrm{mg} / \mathrm{L}$ were defined as high-level resistant. Pearson's $\chi^{2}$ test and Fisher's exact test (when sample size $<5$ ) were used to identify associations between CCs, SCCmec types and resistance levels. A $P$ value for significance was determined by $\alpha$ divided by the number of independent tests performed in a group of comparisons according to Bonferroni's method to minimize the risk of type I error.

\section{Results}

The isolates belonged to CC71 $(n=36)$, CC258 $(n=33)$, CC45 $(n=11)$, CC68 $(n=1)$ and five singleton STs (ST268, ST269, ST284, ST286 and ST431) (Table S1, available as Supplementary data at JAC Online). Thirty-four (94\%) CC71 isolates harboured SCCmecIIIII, which was significantly associated with this CC $(P<0.001)$ and not found in other STs. Thirty-one (94\%) CC258 isolates harboured SCCmecIV, which was significantly associated with this CC $(P<0.001)$. SCCmecIV was also found in three other STs $(268,269$ and 286). SCCmecV was found in four different STs and not significantly associated with any CC. SCCmec was non-typeable by PCR and WGS in 14 isolates, including 10 CC45, 2 CC258 and 2 CC71. CC45 was significantly associated with harbouring a non-typeable SCCmec ( $P<0.001)$. No significant difference was found in the distribution of CCS and SCCmec types between Denmark and the Netherlands, although SCCmecV was found only in Danish isolates $(n=4)$.
As defined for MRSP, all isolates showed MICs at or above the resistance breakpoint of oxacillin ( $R \geq 0.5 \mathrm{mg} / \mathrm{L}$ ). In all SCCmecII-III isolates, oxacillin MICs were high ( $\geq 2 \mathrm{mg} / \mathrm{L}$ ), whereas $77 \%$ of other isolates had low MICs ( $<2 \mathrm{mg} / \mathrm{L}$ ) (Figure 1$)$. SCCmecIV was significantly associated with low-level oxacillin resistance $(P<0.001)$.

All isolates displayed penicillin MICs above the clinical breakpoint $(R>0.25 \mathrm{mg} / \mathrm{L})$. There were no significant differences in penicillin MIC distribution between SCCmec types. Low-level resistance to penicillin (MIC $<8 \mathrm{mg} / \mathrm{L}$ ) was observed in seven isolates, all originating from Denmark $(P=0.001)$.

For ampicillin, 1 isolate tested susceptible (MIC $\leq 0.25 \mathrm{mg} / \mathrm{L}$ ), 15 isolates showed low-level resistance (MIC $0.5-4 \mathrm{mg} / \mathrm{L}$ ) and 70 isolates showed high-level resistance (MIC $>8 \mathrm{mg} / \mathrm{L}$ ). High-level resistance was associated with SCCmecII-III $(P<0.001)$ and lowlevel resistance with SCCmecIV ( $P=0.007)$. Low-level ampicillin resistance was significantly more prevalent in Denmark $(P<0.001)$.

For amoxicillin/clavulanic acid, $80 \%$ of the isolates showed MICs at or above the resistance breakpoint ( $R \geq 0.5 \mathrm{mg} / \mathrm{L})$. Highlevel resistance to amoxicillin/clavulanic acid (MIC $\geq 4 \mathrm{mg} / \mathrm{L}$ ) was significantly associated $(P<0.001)$ with SCCmecII-III of which $68 \%$ had high-level resistance. Susceptibility (MIC $\leq 0.25 \mathrm{mg} / \mathrm{L}$ ) and low-level resistance to amoxicillin/clavulanic acid (MIC $<4 \mathrm{mg} / \mathrm{L}$ ) were seen in $24 \%$ and $68 \%$ of SCCmecIV isolates, in $25 \%$ and $50 \%$ of SCCmecV isolates and in $21 \%$ and $57 \%$ of non-typeable SCCmec isolates, respectively. SCCmecIV was significantly associated with low-level resistance and susceptibility to amoxicillin/clavulanic acid $(P<0.001)$. Low-level resistance was significantly more prevalent in the Netherlands $(P=0.013)$.

Only $30 \%$ (26/86) of the isolates showed MICs above the clinical breakpoint of cefalotin ( $R \geq 4 \mathrm{mg} / \mathrm{L})$. Resistance was primarily seen in SCCmecII-III isolates (65\%). One isolate with SCCmecIV (3\%), three isolates with non-typeable SCCmec types (21\%) and none of the $\mathrm{SCCmecV}$ isolates displayed resistance. The associations of SCCmecII-III with resistance and SCCmecIV with susceptibility were significant $(P<0.001)$.

\section{Discussion}

SCCmecII-III was only present in CC71, whereas SCCmecIV was associated with CC258 but also present in different STs. SCCmecV was shared by different STs. The SCCmec of the majority of CC45 isolates was non-typeable, which correlates with the previously described dissemination of novel SCCmec variants in CC45.,14 These results confirm a link between the SCCmec and CC in MRSP, as previously described. ${ }^{8}$

We found MRSP isolates susceptible to amoxicillin/clavulanic acid and cefalotin across all CCS and SCCmec types. CC71SCCmecII-III isolates showed higher levels of resistance to oxacillin, ampicillin, amoxicillin/clavulanic acid and cefalotin, whereas SCCmecIV was associated with low-level resistance or even susceptibility to several $\beta$-lactams. SCCmecV and non-typeable SCCmec types were not significantly associated with either high- or low-level resistance. Especially for SCCmecV isolates, such lack of statistical association could be due to the low number of isolates representing this type.

The association of SCCmecII-III and high MICs of $\beta$-lactams might have contributed to the success of CC71 globally. MIC differences of $\beta$-lactams between different SCCmec types have been previously reported for $S$. pseudintermedius, with SCCmecII-III 

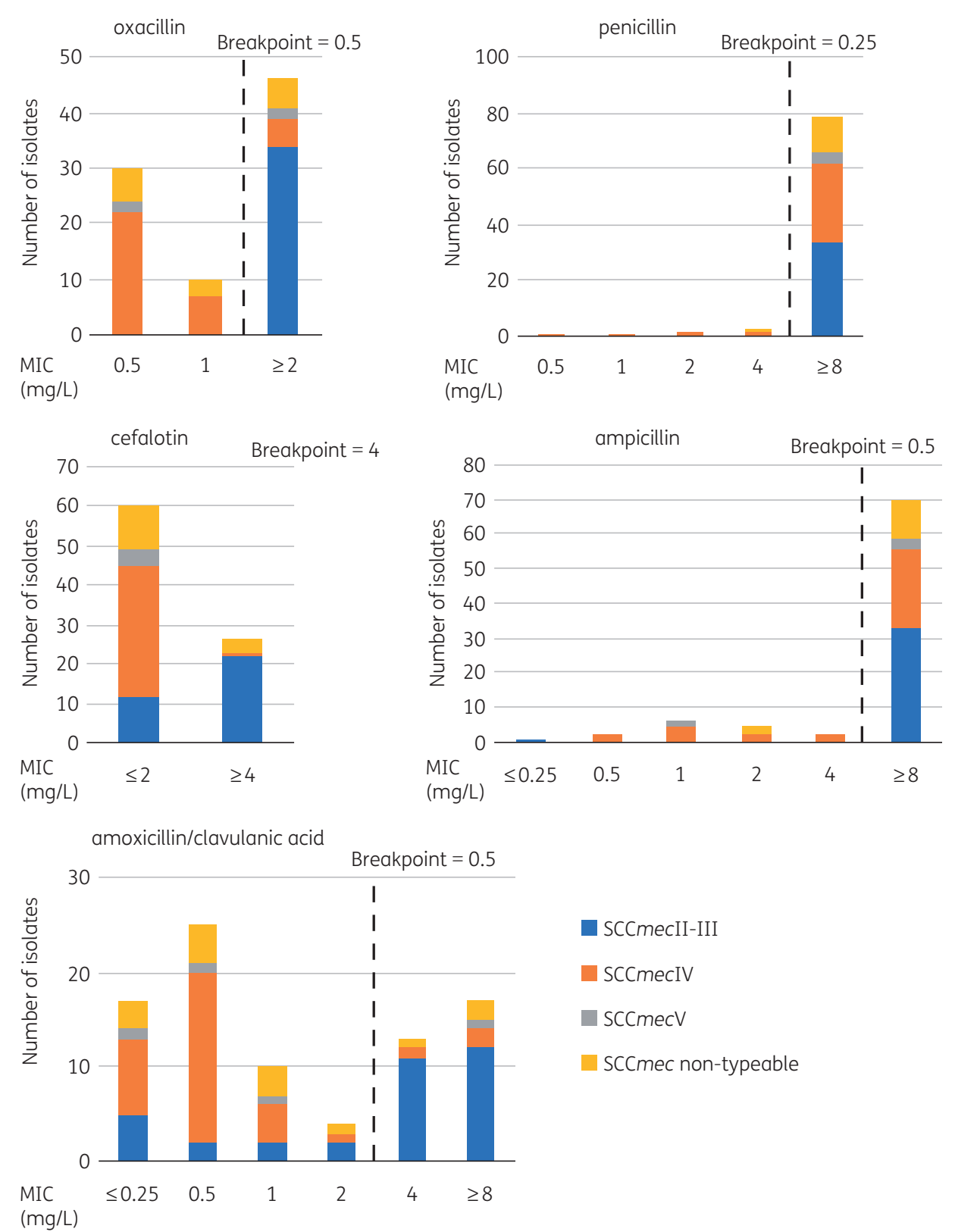

Figure 1. MIC distributions of $\beta$-lactam antibiotics for different SCCmec types. MICs of oxacillin, penicillin, cefalotin, ampicillin and amoxicillin/ clavulanic acid for SCCmecII-III, SCCmecIV, SCCmecV and non-typeable SCCmec types. The broken lines indicate the separation between high- and low-level resistance. This figure appears in colour in the online version of JAC and in black and white in the print version of JAC.

having higher MICs of oxacillin and other $\beta$-lactams than $\mathrm{SCCmecV} .^{7,8}$ This study confirms those findings while expanding the range of antimicrobials tested and the diversity of CCs and SCCmec.

It is unknown how $\mathrm{SCCmec}$ differences influence levels of $\beta$-lactam resistance in MRSP and why there are differences between $\beta$-lactams. In S. aureus, a role of the mecA promoter on the level of PBP2a production and oxacillin resistance level has been shown, ${ }^{15}$ as well as overexpression of blaz resulting in low-level methicillin resistance independently of mecA expression in borderline oxacillin-resistant isolates. ${ }^{16}$ In S. pseudintermedius, mecA (mecI and mecRI) and blaz (blaRI and blaI) regulators are known to modulate expression of mecA. ${ }^{17,18}$ Altogether these studies suggest that the levels of $\beta$-lactam resistance in methicillin-resistant staphylococci are influenced by multiple loci and mechanisms. Further research on sequences, preferably from long-read sequencing, and testing of PBP production and affinity in S. pseudintermedius are required to understand the mechanism responsible for lowlevel $\beta$-lactam resistance in MRSP lineages.

Low-level resistance was significantly more prevalent in Denmark for penicillin and ampicillin, and in the Netherlands for amoxicillin/clavulanic acid. These findings might represent a difference in distribution of isolates between countries, but might also 
be due to methodological differences between the two laboratories or minor variations in MIC affecting the classification of some isolates.

Following EUCAST and CLSI guidelines, oxacillin-resistant isolates should be reported as resistant to all $\beta$-lactams, although clinical evidence is lacking for S. pseudintermedius. The finding of cefalotin and amoxicillin/clavulanic acid susceptibility across all CCs might be clinically relevant, since amoxicillin/clavulanic acid and first-generation cephalosporins are first-line antibiotics for treatment of S. pseudintermedius infections. Cefalotin is highly active against staphylococci and good clinical cure rates have been reported for uncomplicated MRSA skin infections in humans. ${ }^{19}$ For urinary tract infections, anti-MRSA efficacy of high doses of amoxicillin/clavulanic acid has been shown. ${ }^{20}$ For canine urinary tract infections, a higher breakpoint for Staphylococcus spp. exists ( $R$ $>8 \mathrm{mg} / \mathrm{L}) .{ }^{9}$ Using this breakpoint, $90 \%$ of our isolates would be considered susceptible. Notably, the dosing regimens used for firstgeneration cephalosporins and amoxicillin/clavulanic acid in companion animals can be higher than those used for setting clinical breakpoints $(12.5 \mathrm{mg} / \mathrm{kg}$ twice daily for amoxicillin/clavulanic acid and $25 \mathrm{mg} / \mathrm{kg}$ twice daily for cefalotin). It can be hypothesized that MRSP infections caused by isolates with low-level resistance to $\beta$-lactams can be treated by using higher doses and/or more frequent administration, thereby avoiding use of antimicrobials with adverse effects (e.g. rifampicin) or critically important antimicrobials not authorized for veterinary use (e.g. vancomycin). Pharmacokinetic/pharmacodynamic and clinical outcome studies are needed to validate this hypothesis.

\section{Acknowledgements}

This work was presented at the Twenty-Ninth European Congress of Clinical Microbiology \& Infectious Diseases, Amsterdam, The Netherlands, 2019 (Poster P2829) and at the International Conference on One Health Antimicrobial Resistance, Utrecht, The Netherlands, 2019 (Poster P68).

We conducted this study on behalf of the ESCMID Study Group for Veterinary Microbiology (ESGVM), which endorsed the content of the article.

\section{Funding}

This work was supported by the Faculty of Veterinary Medicine, Utrecht University.

\section{Transparency declarations}

None to declare.

\section{Supplementary data}

Table S1 is available as Supplementary data at JAC Online.

\section{References}

1 Pires dos Santos T, Damborg P, Moodley A et al. Systematic review on global epidemiology of methicillin-resistant Staphylococcus pseudintermedius: inference of population structure from multilocus sequence typing data. Front Microbiol 2016; 7: 1599.
2 Duim B, Verstappen KM, Broens EM et al. Changes in the population of methicillin-resistant Staphylococcus pseudintermedius and dissemination of antimicrobial-resistant phenotypes in the Netherlands. J Clin Microbiol 2016; 54: $283-8$.

3 International Working Group on the Classification of Staphylococcal Cassette Chromosome Elements (IWG-SCC). Classification of staphylococcal cassette chromosome mec ( $\mathrm{SCCmec}$ ): guidelines for reporting novel $\mathrm{SCCmec}$ elements. Antimicrob Agents Chemother 2009; 53: 4961-7.

4 Ho C-M, Lin C-Y, Ho M-W et al. Methicillin-resistant Staphylococcus aureus isolates with SCCmec type V and spa types 4437 or t1081 associated to discordant susceptibility results between oxacillin and cefoxitin, Central Taiwan. Diagn Microbiol Infect Dis 2016; 86: 405-11.

5 Chen F-J, Huang I-W, Wang C-H et al. mecA-positive Staphylococcus aureus with low-level oxacillin MIC in Taiwan. J Clin Microbiol 2012; 50: 1679-83.

6 Conceição $\mathrm{T}$, Coelho $\mathrm{C}$, de Lencastre $\mathrm{H}$ et al. Frequent occurrence of oxacillin-susceptible mecA-positive Staphylococcus aureus (OS-MRSA) strains in two African countries. J Antimicrob Chemother 2015; 70: 3200-4.

7 Kasai T, Saegusa S, Shirai M et al. New categories designated as healthcare-associated and community-associated methicillin-resistant Staphylococcus pseudintermedius in dogs. Microbiol Immunol 2016; 60: 540-51.

8 Worthing KA, Schwendener S, Perreten V et al. Characterization of staphylococcal cassette chromosome mec elements from methicillinresistant Staphylococcus pseudintermedius infections in Australian animals. mSphere 2018; 3: e00491-18.

9 CLSI. Performance Standards for Antimicrobial Disk and Dilution Susceptibility Tests for Bacteria Isolated From Animals—Fourth Edition: VET08. 2018.

10 CLSI. Performance Standards for Antimicrobial Disk and Dilution Susceptibility Tests for Bacteria Isolated From Animals-Fifth Edition: VET01. 2018.

11 Wegener A, Broens EM, Zomer A et al. Comparative genomics of phenotypic antimicrobial resistances in methicillin-resistant Staphylococcus pseudintermedius of canine origin. Vet Microbiol 2018; 225: 125-31.

12 Damborg P, Moodley A, Aalbæk B et al. High genotypic diversity among methicillin-resistant Staphylococcus pseudintermedius isolated from canine infections in Denmark. BMC Vet Res 2016; 12: 131.

13 Kaya H, Hasman H, Larsen J et al. SCCmecFinder, a web-based tool for typing of staphylococcal cassette chromosome mec in Staphylococcus aureus using whole-genome sequence data. mSphere 2018; 3: e00612-17.

14 Duim B, Verstappen K, Kalupahana RS et al. Methicillin-resistant Staphylococcus pseudintermedius among dogs in the description of novel SCCmec variants. Vet Microbiol 2018; 213: 136-41.

15 Harrison EM, Ba X, Coll F et al. Genomic identification of cryptic susceptibility to penicillins and $\beta$-lactamase inhibitors in methicillin-resistant Staphylococcus aureus. Nat Microbiol 2019; 4: 1680-91.

16 Hryniewicz MM, Garbacz K. Borderline oxacillin-resistant Staphylococcus aureus (BORSA) - a more common problem than expected? J Med Microbiol 2017; 66: 1367-73.

17 Liu P, Xue H, Wu Z et al. Effect of bla regulators on the susceptible phenotype and phenotypic conversion for oxacillin-susceptible mecApositive staphylococcal isolates. J Antimicrob Chemother 2016; 71: 2105-12.

18 Black CC, Eberlein LC, Solyman SM et al. The role of mecA and blaZ regulatory elements in mecA expression by regional clones of methicillin-resistant Staphylococcus pseudintermedius. Vet Microbiol 2011; 151: 345-53.

19 Chen AE, Carroll KC, Diener-West M et al. Randomized controlled trial of cephalexin versus clindamycin for uncomplicated pediatric skin infections. Pediatrics 2011; 127: e573-80.

20 Guignard B, Entenza JM, Moreillon P. $\beta$-Lactams against methicillinresistant Staphylococcus aureus. Curr Opin Pharmacol 2005; 5: 479-89. 